\title{
Neuronal eotaxin and the effects of CCR3 antagonist on airway hyperreactivity and $\mathrm{M} 2$ receptor dysfunction
}

\begin{abstract}
Allison D. Fryer, ${ }^{1,2,3}$ Louis H. Stein, ${ }^{3}$ Zhenying Nie,, ${ }^{2}$ Damian E. Curtis, ${ }^{2}$ Christopher M. Evans, ${ }^{3}$ Simon T. Hodgson, ${ }^{4}$ Peter J. Jose, ${ }^{5}$ Kristen E. Belmonte, ${ }^{6}$ Erin Fitch, ${ }^{2}$ and David B. Jacoby ${ }^{1,2,7}$

1Division of Pulmonary and Critical Care Medicine and 2Department of Physiology and Pharmacology, Oregon Health and Science University, Portland, Oregon, USA. ${ }^{3 B}$ loomberg School of Public Health, Johns Hopkins University, Baltimore, Maryland, USA. ${ }^{4}$ GlaxoSmithKline, Stevenage, United Kingdom. ${ }^{5}$ Leukocyte Biology Section, Imperial College, London, United Kingdom. ${ }^{6}$ GlaxoSmithKline, King of Prussia, Pennsylvania, USA. ${ }^{7}$ School of Medicine,
\end{abstract} Johns Hopkins University, Baltimore, Maryland, USA.

\begin{abstract}
Eosinophils cluster around airway nerves in patients with fatal asthma and in antigen-challenged animals. Activated eosinophils release major basic protein, which blocks inhibitory M2 muscarinic receptors (M2Rs) on nerves, increasing acetylcholine release and potentiating vagally mediated bronchoconstriction. We tested whether GW701897B, an antagonist of CCR3 (the receptor for eotaxin as well as a group of eosinophil active chemokines), affected vagal reactivity and M2R function in ovalbumin-challenged guinea pigs. Sensitized animals were treated with the CCR3 antagonist before inhaling ovalbumin. Antigen-challenged animals were hyperresponsive to vagal stimulation, but those that received the CCR3 antagonist were not. M2R function was lost in antigen-challenged animals, but not in those that received the CCR3 antagonist. Although the CCR3 antagonist did not decrease the number of eosinophils in lung tissues as assessed histologically, CCR3 antagonist prevented antigen-induced clustering of eosinophils along the nerves. Immunostaining revealed eotaxin in airway nerves and in cultured airway parasympathetic neurons from both guinea pigs and humans. Both IL-4 and IL-13 increased expression of eotaxin in cultured airway parasympathetic neurons as well as in human neuroblastoma cells. Thus, signaling via CCR3 mediates eosinophil recruitment to airway nerves and may be a prerequisite to blockade of inhibitory M2Rs by eosinophil major basic protein.
\end{abstract}

\section{Introduction}

In the airways, neuronal M2 muscarinic receptors (M2Rs) limit vagally induced bronchoconstriction by inhibiting acetylcholine release from parasympathetic nerve endings (1). These receptors, which are present in human (2) as well as animal airways, are dysfunctional after antigen inhalation in animals (3). Loss of M2R function increases acetylcholine release and potentiates vagally mediated bronchoconstriction. These receptors are also dysfunctional in some patients with asthma (4).

Eosinophils are clustered along the airway nerves in antigenchallenged animals and in patients with asthma (5). Activation of eosinophils releases major basic protein (MBP), an antagonist at the M2Rs (6). By blocking the M2Rs, MBP eliminates the normal negative feedback regulation of acetylcholine release, increasing vagally mediated bronchoconstriction (7). In antigen-challenged animals, depleting eosinophils using an antibody against IL-5 (8), or neutralizing MBP with specific antibody (7) or with polyanionic substances such as heparin or poly-L-glutamic acid (3), prevents antigen-induced M2R dysfunction and airway hyperreactivity.

We have previously shown that recruitment of eosinophils to the airway nerves is critical in the development of M2R dysfunction. The number of eosinophils in contact with airway nerves, but not the total number of eosinophils in the airway tissues or in lung

Nonstandard abbreviations used: MBP, eosinophil major basic protein; M2R, M2 muscarinic receptor; PGP 9.5, protein gene product 9.5 .

Conflict of interest: The authors have declared that no conflict of interest exists.

Citation for this article: J. Clin. Invest. 116:228-236 (2006)

doi:10.1172/JCI25423. lavage fluid, correlates with the degree of M2R dysfunction (5). Dexamethasone, at a dose that does not decrease the total number of eosinophils in the lungs or the airways of antigen-challenged animals, prevents eosinophil accumulation around the airway nerves (9). This is sufficient to prevent M2R dysfunction and airway hyperreactivity in these animals.

Eotaxin is a chemokine produced by a wide variety of cells. It is found in the airway lavage fluid of antigen-challenged animals and in humans with asthma $(10,11)$. Eotaxin signals exclusively via the CCR3 receptor, mediating eosinophil chemotaxis and activation (12). In this study, we tested whether eotaxin is present in airway parasympathetic neurons and whether signaling via CCR3 mediates eosinophil migration to airway nerves.

\section{Results}

Effect of CCR 3 antagonist on antigen-induced airway hyperreactivity. To test the effect of signaling via CCR3 in antigen-induced airway hyperreactivity, we sensitized guinea pigs by i.p. injection of ovalbumin and subsequently challenged them with inhaled ovalbumin aerosol (3). The day after inhalational challenge, animals were anesthetized, paralyzed, and ventilated via a tracheostomy. Electrical stimulation of the vagus nerves caused frequency-dependent bronchoconstriction that was increased in antigen-challenged animals (Figure 1). This was prevented by treatment of animals with the CCR3 antagonist GW701897B. Bronchoconstriction induced by i.v. acetylcholine or methacholine was not different among control, antigen-challenged, or CCR3 antagonist-treated groups, demonstrating that smooth muscle contractile function was not increased (Figure 2). Thus, in antigen-challenged animals, hyper- 


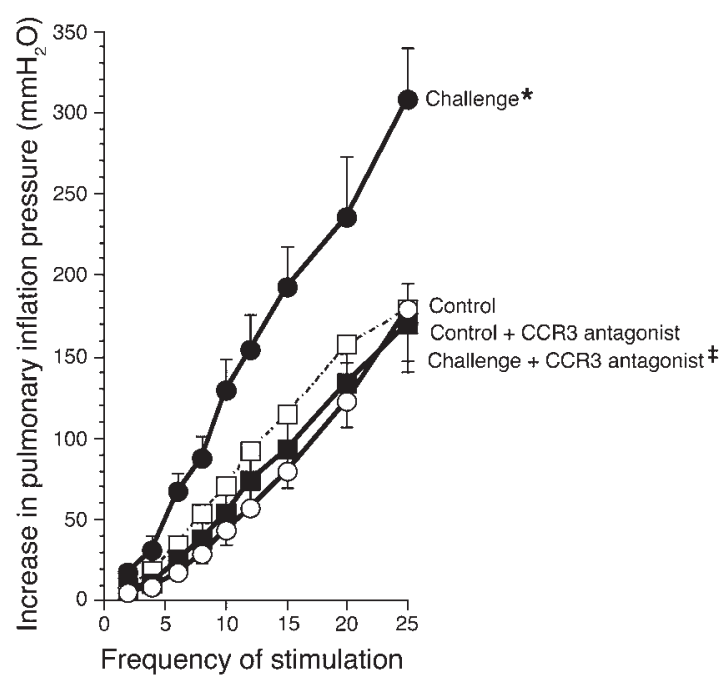

reactivity was mediated by increased release of acetylcholine from parasympathetic nerve endings, and hyperreactivity was inhibited by pretreatment with the CCR3 antagonist.

Effect of CCR 3 antagonist on antigen-induced M2R dysfunction. In control animals, stimulating M2Rs on the nerves using the muscarinic agonist pilocarpine (1-100 $\mu \mathrm{g} / \mathrm{kg}$, i.v.) inhibited vagally mediated bronchoconstriction in a dose-dependent fashion. This demonstrated the intact function of the M2R. In contrast, in antigenchallenged animals, pilocarpine did not inhibit vagally mediated bronchoconstriction (Figure 3). Thus, antigen-induced hyperreactivity was accompanied by M2R dysfunction. Both hyperreactivity and M2R dysfunction were prevented by the CCR3 antagonist.

Effects of CCR 3 antagonist on antigen-induced inflammation. Antigen challenge increased total leukocytes in lung lavage (Figure 4). This increase was composed primarily of macrophages and eosinophils and was prevented by the CCR3 antagonist.

Antigen challenge increased the total number of eosinophils in airway tissues as assessed histologically. This increase in eosinophils was not significantly attenuated by the CCR3 antagonist (Figures 5 and 6). Antigen challenge also increased the number of eosinophils around the airway nerves. Accumulation of eosinophils around the nerves, but not the influx of eosinophils into the rest of the airway, was prevented by the CCR3 antagonist (Figures 5 and 6).

Eotaxin expression in airway nerves. Immunostaining with antibody against guinea pig eotaxin showed positive staining in airway epithelium, smooth muscle, and nerves (Figure 7). Antigen challenge increased eotaxin staining in all 3 tissues (Figure 7, D-J). Eotaxin-1 was also expressed in airway nerves from a nonasthmatic human (Figure 8), demonstrating that neuronal expression of eotaxin is not restricted to guinea pigs. Nerve cells isolated from human tracheas also stained positively for eotaxin (Figure 9A). This is the first report to our knowledge that parasympathetic nerves could be isolated from human tissue; therefore, we also confirmed that the cells were nerve cells by demonstrating that they could be labeled with antibody against protein gene product 9.5 (PGP 9.5), a marker for nerves (Figure 9C).

$I L-4$ receptor on cultured airway parasympathetic nerves and buman neuroblastoma cells. Because both human and guinea pig airway parasympathetic nerves express eotaxin, we sought to determine whether eotaxin expression in neurons could be regulated by IL-4 and IL-13. These studies were carried out in primary cultures of

\section{Figure 1}

Vagally mediated bronchoconstriction in control and antigen-challenged animals. Antigen challenge induced potentiation of vagally induced bronchoconstriction is inhibited by the CCR3 antagonist. In control animals (open circles), stimulation of both vagi (10 V, 0.2millisecond pulse duration, 5-second pulse train) caused frequencydependent bronchoconstriction that was significantly increased in antigen-challenged animals (filled circles). Pretreatment with the CCR3 antagonist (filled squares) prevented the increase in vagally induced bronchoconstriction in antigen-challenged guinea pigs. $n=5-7$. *Significantly different from control, $P=0.001$; $\neq$ significantly different from challenged, $P=0.0006$. The CCR3 antagonist did not affect vagally induced bronchoconstriction in non-antigen-challenged control animals (open squares; $n=6$ ).

guinea pig nerves and in human neuroblastoma cells, since these cells are more readily available than primary human nerve cells. Prior to these studies, we demonstrated that guinea pig primary nerve cell cultures and human neuroblastoma cells express IL-4 receptor, as assessed by immunostaining (Figure 10).

Effects of IL-4 and IL-13 on eotaxin expression in primary cultures of airway parasympathetic neurons. Primary cultures of guinea pig airway parasympathetic neurons stained positive for eotaxin (Figure 11, A and B). To determine whether IL-4 and IL-13, which may be produced during allergic responses, might be involved in increasing eotaxin expression by airway nerves, we treated cultured guinea pig parasympathetic neurons with IL-4 and IL-13 $(1 \mathrm{ng} / \mathrm{ml})$ for 8 hours. Staining was increased by treatment with IL-4 (Figure 11, $\mathrm{C}$ and D). MetaMorph analysis of images revealed a $60 \%$ increase

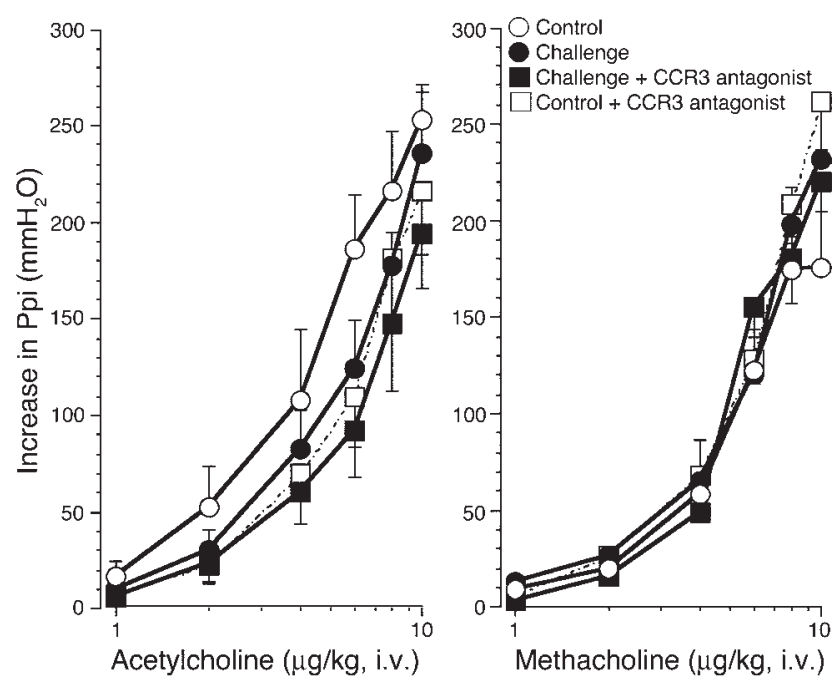

\section{Figure 2}

Bronchoconstriction in response to exogenous cholinergic agonists. Bronchoconstriction induced by i.v. acetylcholine (left) or methacholine (right) in vagotomized animals was not changed by antigen challenge (filled circles) or by treatment of antigen-challenged animals with the CCR3 antagonist (filled squares). $n=5-7$. The CCR3 antagonist did not affect bronchoconstriction in non-antigen-challenged control animals (open squares; $n=5$ ). Ppi, pulmonary inflation pressure. 


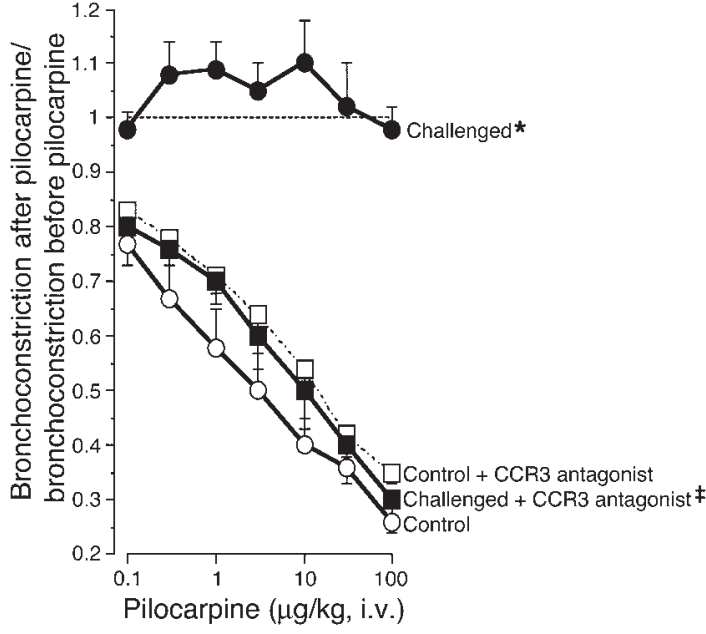

in staining intensity. Similar results were obtained after treatment with IL-13 (data not shown).

Regulation of eotaxin expression in buman neuroblastoma cells by IL-4 and IL-13. Treating neuroblastoma cells with IL-4 $(0.1-10 \mathrm{ng} / \mathrm{ml})$ increased eotaxin mRNA and protein expression in a concentrationdependent fashion, with a maximal effect seen at an IL-4 concentration of $1 \mathrm{ng} / \mathrm{ml}$ (data not shown). Treatment of neuroblastoma cells with IL-4 at this concentration increased eotaxin-1 mRNA as assessed by real-time RT-PCR, with a peak increase occurring about 2 hours after stimulation (Figure 12). eotaxin-3 mRNA was also detectable by RT-PCR but was not induced by IL-4 (data not shown). eotaxin-2 was not expressed by these cells. Eotaxin protein expression, assessed by immunostaining, was also increased, with a peak increase coming after 8 hours (Figure 12). Similar results were obtained with IL-13, which increased eotaxin mRNA $2.6 \pm 0.29$ fold $(P<0.001 ; n=4)$ and eotaxin immunostaining $2.4 \pm 0.41$-fold in neuroblastoma cells, with a maximal effect seen at $1 \mathrm{ng} / \mathrm{ml}$. Likewise, IL-13 increased eotaxin immunostaining in primary cultures of guinea pig airway parasympathetic neurons by $63 \% \pm 16 \%$ $(P=0.002 ; n=4$; data not shown).

Adhesion of eosinophils to cultured airway parasympathetic neurons. To determine whether eotaxin might be a prerequisite for eosinophil adherence to parasympathetic nerve fibers, we isolated guinea pig eosinophils by peritoneal lavage and purified them by centrifugation over a Percoll gradient. Eosinophils were incubated with cultured guinea pig parasympathetic neurons for 30 minutes, after which nonadherent eosinophils were washed off. As we have previously shown (13), treating cultured parasympathetic neurons with TNF- $\alpha$ and IFN- $\gamma$ increased eosinophil adherence. This is the result of increased ICAM-1 expression by the nerves (13). Treating these cells with the CCR3 antagonist prevented the increased adherence of eosinophils to these neurons (Figure 13).

\section{Figure 4}

Inflammatory cells in lung lavage fluid. Antigen challenge caused a nonsignificant increase in total leukocytes, which consisted primarily of macrophages and eosinophils. This was prevented by the CCR3 antagonist. $n=5-7$. ₹Significantly different from control, $P<0.05$; *significantly different from challenged, $P<0.05$. BAL, bronchoalveolar lavage.

\section{Figure 3}

CCR3 antagonist prevents antigen-induced M2R dysfunction. Pilocarpine inhibited vagally induced bronchoconstriction in control animals (open circles) demonstrating functional M2Rs. Pilocarpine did not inhibit vagally induced bronchoconstriction in antigen-challenged animals (filled circles), demonstrating that the M2Rs were no longer responding to agonist stimulation. M2R dysfunction was prevented by the CCR3 antagonist (filled squares). $n=5-7$. ${ }^{*}$ Significantly different from control, $P=0.0006$; : significantly different from challenged, $P=0.001$. The CCR3 antagonist did not affect the response to pilocarpine in non-antigen-challenged control animals (open squares; $n=5$ ).

\section{Discussion}

We have previously shown that antigen sensitization, even in the absence of antigen challenge, causes eosinophils to migrate to the airway nerves (14), yielding a histological picture similar to that seen in human asthma (5). Subsequent activation of these nerveassociated eosinophils in response to antigen challenge (7) or viral infections (15) causes release of eosinophil MBP, an allosteric antagonist at M2Rs. Blocking these receptors on the parasympathetic nerves increases the release of acetylcholine, potentiating vagally mediated reflex bronchoconstriction (16).

The data presented in this paper demonstrate that eotaxin is present in the airway nerves of both guinea pigs and humans, extending the recent observation that eotaxin is expressed in nerves of dust mite-exposed rhesus monkeys (17). In vivo, blocking CCR3, the sole receptor for eotaxin, prevents antigen-induced migration of eosinophils to the airway nerves and prevents antigen challenge from causing M2R dysfunction and airway hyperreactivity.

Airway parasympathetic nerves, as well as human neuroblastoma cells (SK-N-SH cells), express the receptor for IL-4 (Figure 10).

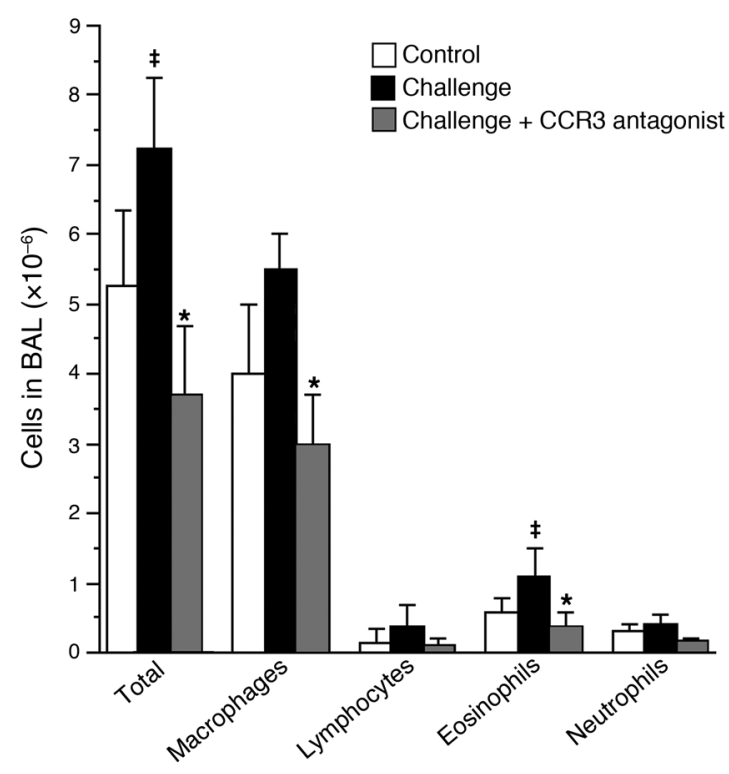




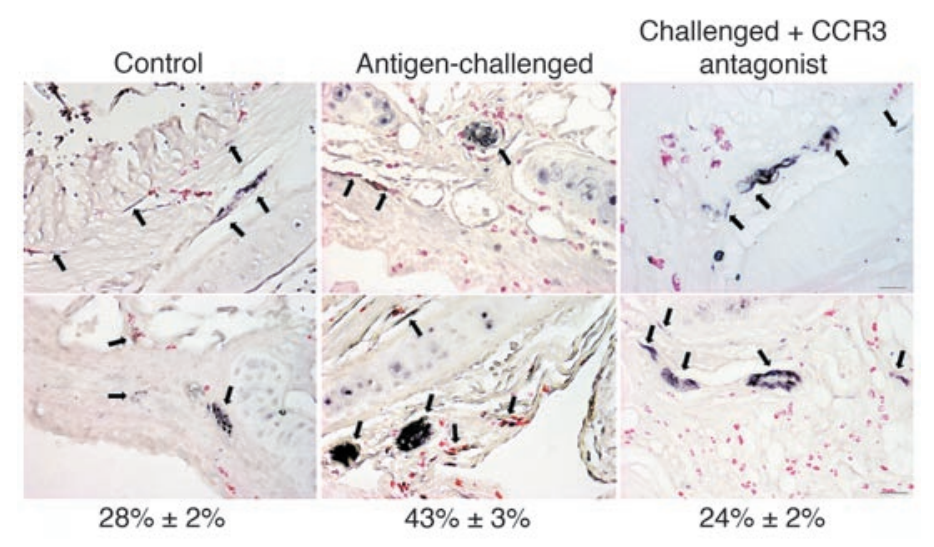

\section{Figure 5}

Treatment with a CCR3 antagonist prevents the increase in eosinophils around airway nerves after antigen challenge. Airway nerves (arrows) were stained black with antibody against PGP 9.5, and eosinophils were stained red with chromotrope $2 \mathrm{R}$ in control and antigen-challenged animals and in antigen-challenged animals treated with CCR3 antagonist. Numbers under the panels refer to the percentage of eosinophils (mean \pm SEM; $n=5-7$ ) in contact with airway nerves. The scale bar of $50 \mu \mathrm{m}$ applies to all panels except the one in the upper right, where it equals $20 \mu \mathrm{m}$.
Treatment with this Th 2 cytokine increased neuronal expression of eotaxin (Figures 11 and 12), results that may be relevant to the role of eotaxin in antigen-challenged airways. IL-4-induced eotaxin expression in SK-N-SH cells was limited to eotaxin-1. Eotaxin-2 was not expressed by these cells even after treatment with IL-4; although eotaxin-3 was expressed by these neuroblastoma cells, it was not induced by treatment with IL-4 (data not shown).

Eotaxin is also expressed by a wide variety of other tissues in the airways, including smooth muscle and epithelium (12). Blocking CCR3 not only protected the M2R but also decreased the antigeninduced influx of eosinophils into the lung lavage fluid. Despite this decrease in eosinophil migration into the lavage, overall eosinophil number in the airway tissues was not significantly decreased by blocking CCR3. However, the CCR3 antagonist prevented eosinophil accumulation specifically around the airway nerves and by so doing prevented antigen-induced M2R dysfunction and airway hyperreactivity.

The accumulation of eosinophils around airway nerves is critical to the physiological response to a variety of airway insults. We have previously demonstrated that recruitment of eosinophils to the lungs and nerves occurs independently of activation. Sensitization without antigen challenge is sufficient to recruit eosinophils to the nerves without any physiological changes that would suggest activation of the eosinophils (15). Once the eosinophils are in contact with the nerves, they can be activated by antigen challenge (3), viral infection (15), or ozone inhalation $(18,19)$. In each case, the result is M2R dysfunction mediated by MBP (7). The data here demonstrate in vivo and in vitro that eosinophil recruitment to nerves is mediated by a CCR3 agonist. In contrast, eosinophil recruitment to the lungs is not dependent upon a CCR3 agonist. A concentration of the CCR3 antagonist sufficient to prevent hyperreactivity and protect neuronal M2R function (Figures 1 and 3) did not decrease the number of eosinophils within the lungs, although it did decrease the number associated with the nerves (Figures 5 and 6).

We have previously shown that dexamethasone treatment has effects similar to those of the CCR3 antagonist in the current study. Therefore, M2R function is preserved in antigen-challenged animals treated with dexamethasone. Tissue sections from antigen-challenged animals also revealed that eosinophil number is not different between animals treated or not treated with dexamethasone. However, histological examination revealed that dexamethasone, like the CCR3 antagonist in the current study, prevented eosinophils from accumulating around airway nerves and, in so doing, prevented antigen-induced hyperreactivity (9). Whether this effect of dexamethasone was due to suppression of eotaxin expression by the nerves or decreased CCR3 expression by the eosinophil, or whether unrelated mechanisms are important, is unknown.

The role of eosinophils as effector cells in asthma has been debated in recent years. A study in patients with asthma examined the effects of neutralizing IL-5 with a humanized monoclonal antibody and suggested that substantial decreases in blood and sputum eosinophilia could occur without a beneficial effect on airway reactivity (20). However, histological examination of airway biopsies from these subjects showed only modest decreases in tissue eosinophils and little or no decrease in tissue MBP (21). Likewise, studies of the role of eosinophils in mouse models of allergic airway disease have yielded conflicting results, with some studies showing a crucial role for eosinophils in airway hyperreactivity (22,
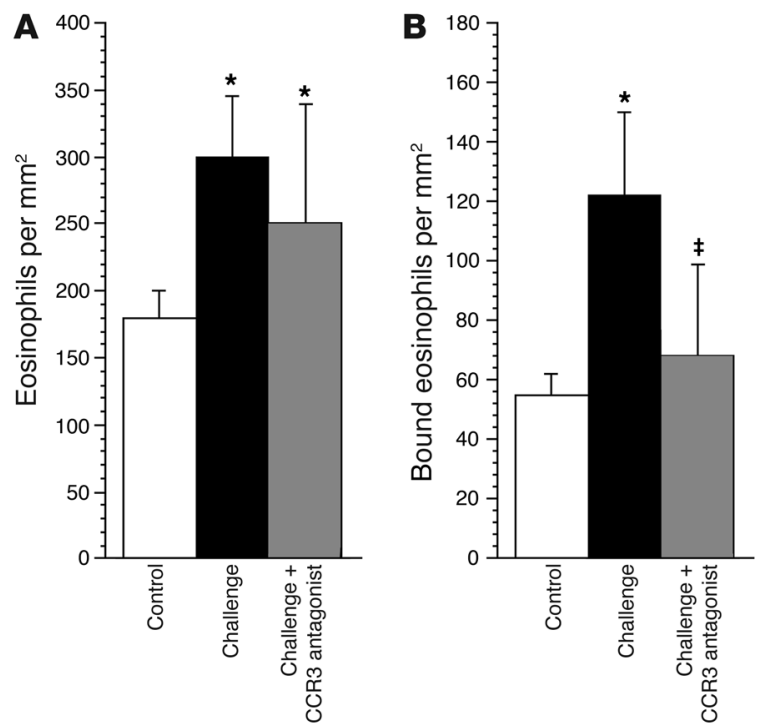

\section{Figure 6}

Antigen challenge increases eosinophils in airway tissues in general (A) as well as specifically in the area of airway nerves (B). The CCR3 antagonist did not prevent the general increase in airway tissue eosinophils (A). In contrast, the CCR3 antagonist selectively prevented the antigen-induced increase in eosinophils around the nerves (B). $n=5-7$. *Significantly different from control, $P<0.01$; ₹significantly different from challenged, $P<0.01$. 

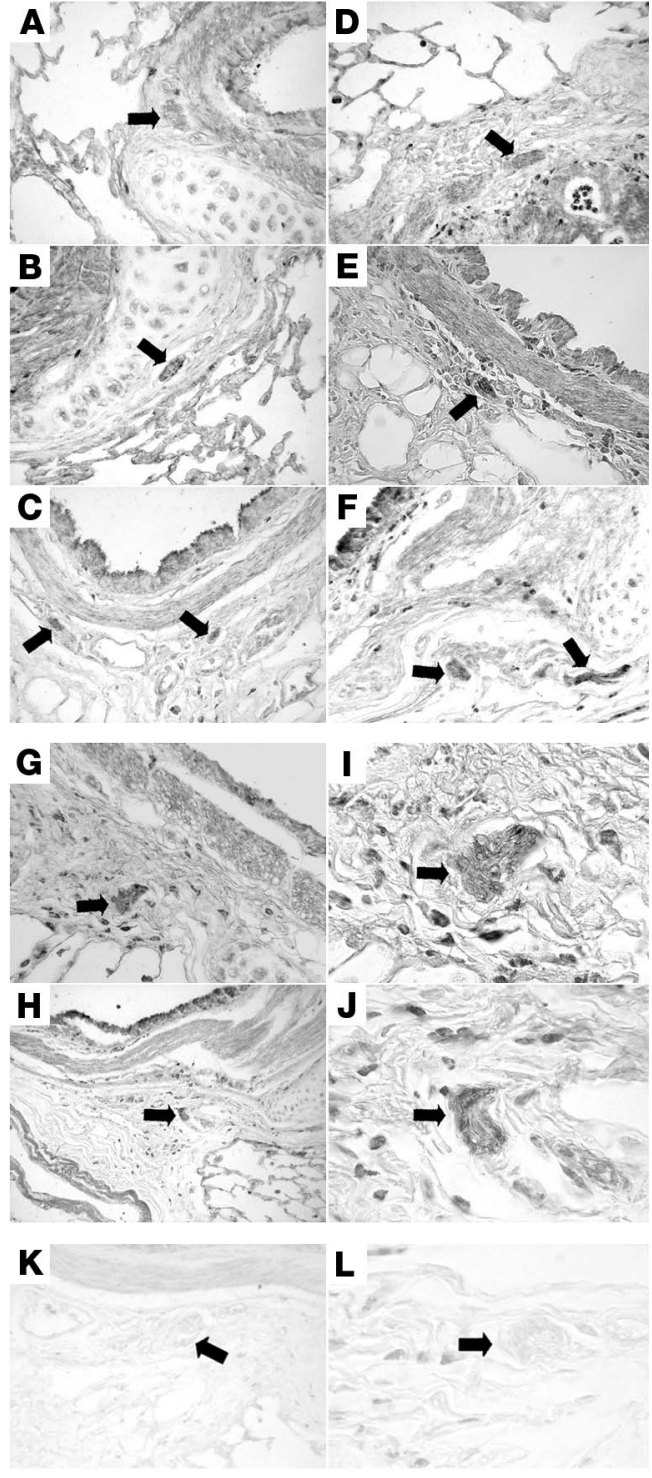

23) and others suggesting that the eosinophil is more important in airway remodeling (24).

Eotaxin is likely to be involved in the pathogenesis of asthma. Expression of both eotaxin and CCR3 is increased in bronchial biopsies from patients with asthma (25). Furthermore, the number of eotaxin-positive cells in the airway correlates with airway hyperreactivity and with asthma symptom scores (25).

Eotaxin binding to CCR3 activates eosinophils, as indicated by increased calcium influx, increased CD11 and CD18 expression, ERK and p38 MAPK activation, increased production of reactive oxidant species, actin polymerization, and degranulation $(12,26)$. Increased expression of CD11 and CD18 is likely to be relevant to the interaction of eosinophils with airway nerves, as we have previously shown that binding of eosinophils to primary cultures of airway parasympathetic nerves is prevented by a blocking antibody against ICAM, the adhesion molecule on the neurons to which the CD11/CD18 complex on the eosinophil binds (13). Moreover, ligation of the CD11/CD18 complex on the eosinophil facilitates eosinophil activation (27). Activation of eosinophils with subse-

\section{Figure 7}

Immunostaining of airway tissues for eotaxin. Eotaxin expression was present throughout the airways, including in the nerves (arrows), of control guinea pigs $(\mathbf{A}-\mathbf{C})$ and was increased after antigen challenge (D-J). Staining of nerves (assessed as mean pixel intensity) was increased from $64.8 \pm 5.9$ in controls to $114.3 \pm 7.8$ in antigenchallenged animals $(P=0.002)$. Nerve bundles in antigen-challenged guinea pigs are shown under low $(\mathbf{G}$ and $\mathbf{H})$ and high magnification ( $\mathbf{I}$ and $\mathbf{J}$ ). There was no positive eotaxin stain in lungs or nerves (arrows) of either control (K) or antigen-challenged (L) lung when preimmune serum was used in place of the antibody. Scale bars: $50 \mu \mathrm{m}$.

quent degranulation releases MBP, which blocks the M2R on the nerve, increasing release of acetylcholine (7).

We found that eotaxin expression in human neuroblastoma cells is potently induced by IL-4 and IL-13. This is consistent with findings in fibroblasts (28) and suggests that Th2 lymphocytes may regulate the neural expression of eotaxin in the airways of patients with asthma. Immunostaining of airway tissues from antigenchallenged guinea pigs showed increased eotaxin staining in the airway nerves. In addition to eosinophils, CCR3 is also expressed on basophils, mast cells, and some Th2 lymphocytes (12). The contribution of the effects of a CCR3 antagonist on these cells to the decrease in localization of eosinophils to the airway nerves is not known. However, production of IL- 4 and IL-13 by these cells might amplify the expression of eotaxin by the airway nerves, thereby indirectly increasing eosinophil localization and activation.

In addition to eotaxin, several other chemokines bind to CCR3 and may participate in the interactions we have described. These include eotaxin-2 and -3 (12), monocyte chemoattractant protein-2, -3 , and -4 (29), RANTES (29), CCL15 (30), and CCL28 (31). The role of these chemokines in the interaction of eosinophils with nerves will depend upon their efficacy as CCR3 agonists, as well as the extent and timing of their production by neurons and other cells.

Eosinophil adhesion to airway parasympathetic neurons in culture was also inhibited by the CCR3 receptor antagonist. The mechanism of this effect is not known but might reflect a decreased activation of eosinophil adhesion molecules in the absence of eotaxin, as has been shown for CD11 (32), which we have shown is required for adhesion of eosinophils to parasympathetic nerves (33). Moreover, stimulation of CCR3 by eotaxin has been shown to change eosinophil integrin function such that the eosinophil develops a preference for binding to ICAM-1 rather than VCAM $(34,35)$. As we have shown that binding to ICAM-1
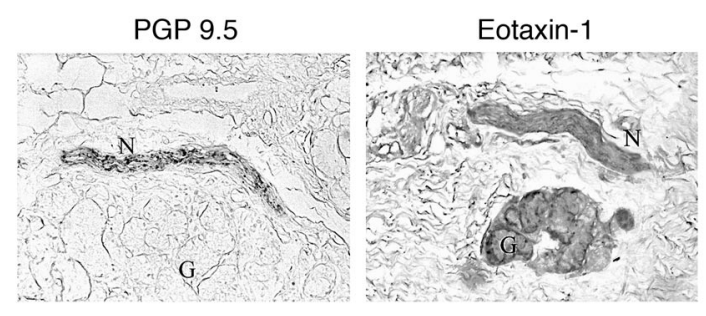

\section{Figure 8}

Immunostaining of human airway nerves for eotaxin. Airway nerves were labeled with an antibody against PGP 9.5 (a marker of nerves; left panel) and an adjacent section labeled with antibody against human eotaxin (right panel). The eotaxin antibody labeled nerves $(\mathrm{N})$ and glands $(G)$ in a nonasthmatic individual. Scale bar: $10 \mu \mathrm{m}$. 

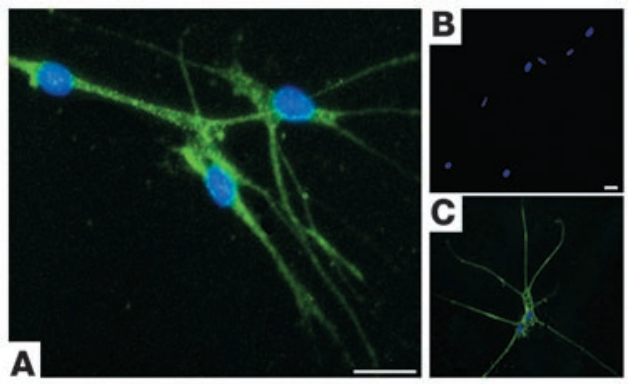

mediates eosinophil adherence to primary cultures of airway parasympathetic neurons (33), preventing the CCR3-mediated switch to ICAM-1 binding could contribute to the lack of binding in the absence of CCR 3 stimulation. Interference with this interaction might also contribute to the redistribution of eosinophils away from nerves in animals treated with the CCR3 antagonist in vivo.

It has been shown that the M2Rs in the airways are dysfunctional in some patients with allergic asthma (4). We have previously shown that antigen-induced M2R dysfunction is mediated by MBP, an endogenous antagonist for $\operatorname{M} 2 \mathrm{Rs}(3,6,7)$. The data presented in this article support CCR3 as a potential drug target in the treatment of asthma. Migration of eosinophils to the airway nerves and subsequent loss of neuronal M2R function and airway hyperreactivity were all prevented when the CCR3 receptors were blocked, suggesting that this treatment may be beneficial in asthma attacks resulting from the activation of nerve-associated eosinophils.

\section{Methods}

Animals. Specific pathogen-free female Dunkin-Hartley guinea pigs (250-300 g) were purchased from Hilltop Animal Farms. All animals were shipped in filtered crates and kept in high-efficiency particulate-filtered air. Guinea pigs were fed a normal diet (Prolab; Agway) and were handled in accordance with the standards established by the US Animal Welfare Acts set forth in NIH guidelines and the "Policy and Procedures Manual" published by the Johns Hopkins University School of Hygiene and Public Health Animal Care and Use Committee. Protocols were reviewed and approved by the institutional animal care and use committees of both Johns Hopkins University and the Oregon Health and Science University.

Sensitization and challenge with antigen. Guinea pigs were sensitized by injection with ovalbumin (Sigma-Aldrich) every other day for a total of 3 injections (10 mg/kg, i.p.), as previously described (3). Three weeks after the last ovalbumin injection, sensitized animals were challenged with an aerosol of $5 \%$ ovalbumin for 5 minutes or until signs of respiratory distress appeared, in which case antigen challenge was immediately stopped.

At the end of the experiments, sensitization was confirmed in some randomly chosen animals by injection of ovalbumin $(1.0 \mathrm{mg} / \mathrm{kg}$ i.v. $)$. This dose of ovalbumin caused acute anaphylaxis in all sensitized animals regardless of dexamethasone pretreatment, but it had no effect in nonsensitized controls.

\section{Figure 10}

Immunostaining for IL-4 receptor. IL-4 receptors were present on primary cultures of guinea pig airway parasympathetic neurons $(\mathbf{A})$ and on human neuroblastoma cells (B). Neurons were stained with antibody against IL-4 (green) and DAPI, which stains the nuclei blue. Insets show cells in the absence of primary antibody; only blue DAPI-stained nuclei are visible (10-fold lower magnification). Scale bars: $30 \mu \mathrm{m}$.

\section{Figure 9}

Primary cultures of human airway parasympathetic nerves express eotaxin. Neurons are stained with antibody against eotaxin (A), no primary antibody (B), or with antibody against the nerve-specific protein PGP 9.5 (C). All cells are stained with DAPI, labeling nuclei blue. Scale bars: $25 \mu \mathrm{M}$.

Treatments. Some guinea pigs were pretreated with the CCR3 antagonist GW701897B (5 mg/kg, i.p.; GlaxoSmithKline) 1 hour before antigen challenge. This compound has been tested against a panel of receptors including, but not limited to, CCR1, CCR2, and CXCR2 and was found to be more than 100 -fold selective for CCR3. Plasma $t_{1 / 2}$ in the guinea pig is 1.4 hours. Characteristics of GW701897B were established in tests by GlaxoSmithKline (Stevenage, United Kingdom).

Measurements of pulmonary inflation pressure. Experiments were conducted 18-24 hours after antigen challenge. The guinea pigs were anesthetized with urethane (1.8 g/kg, i.p.; Sigma-Aldrich). This dose produces a deep anesthesia lasting $8-10$ hours, although none of these experiments lasted longer than 4 hours.

Physiological studies were performed as previously described (3). Briefly, the jugular veins were cannulated bilaterally for the administration of drugs, and 1 internal carotid artery was cannulated for measurement of blood pressure and heart rate. The trachea was cannulated, and the animals were mechanically ventilated at a respiratory rate of 100 breaths/ minute with the tidal volume of $10 \mathrm{ml} / \mathrm{kg}$. The animals were paralyzed by continuous succinylcholine infusion $(10 \mu \mathrm{g} / \mathrm{kg} / \mathrm{min}$, i.v.). Pulmonary inflation pressure (Ppi) was measured via a sidearm off the tracheal cannula. Bronchoconstriction was measured as the increase in Ppi above the basal inflation pressure produced by the ventilator.

Vagal reactivity. Anesthetized, ventilated, and paralyzed guinea pigs were vagotomized. The distal portions of the cut vagi were stimulated electrically via platinum electrodes. Electrical stimulation of the vagus nerves (1-15 Hz, $10 \mathrm{~V}, 0.2$-ms pulse duration, for 5 seconds at 90-second intervals) produced frequency-dependent bronchoconstriction and bradycardia that recovered on cessation of stimulation. Atropine was given at the end of the experiments to confirm that vagally induced bronchoconstriction in these experiments was cholinergic.

Neuronal M2R function. Vagi were stimulated electrically $(15 \mathrm{~Hz}, 0.2-\mathrm{ms}$ pulse duration, for 5 seconds at 60 -second intervals) with voltage adjusted to produce repeated bronchoconstrictions of similar magnitude before administration of the muscarinic agonist pilocarpine. Cumulative doses of pilocarpine (0.1-100 $\mu \mathrm{g} / \mathrm{kg}$, i.v.; Sigma-Aldrich) were then administered, and the effects on vagally induced bronchoconstriction were measured. Pilocarpine stimulates the M2Rs, inhibiting vagally induced bronchoconstriction. The degree to which pilocarpine inhibits vagally induced bronchoconstriction is therefore a measure of M2R function.
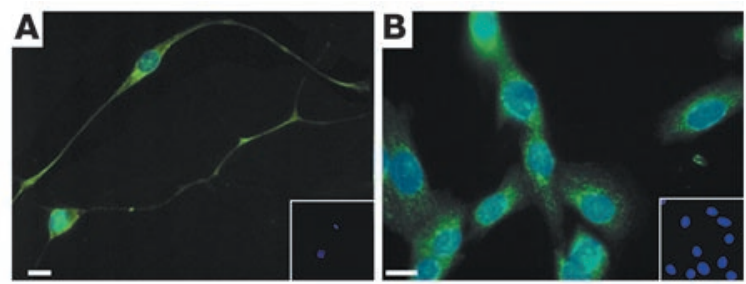


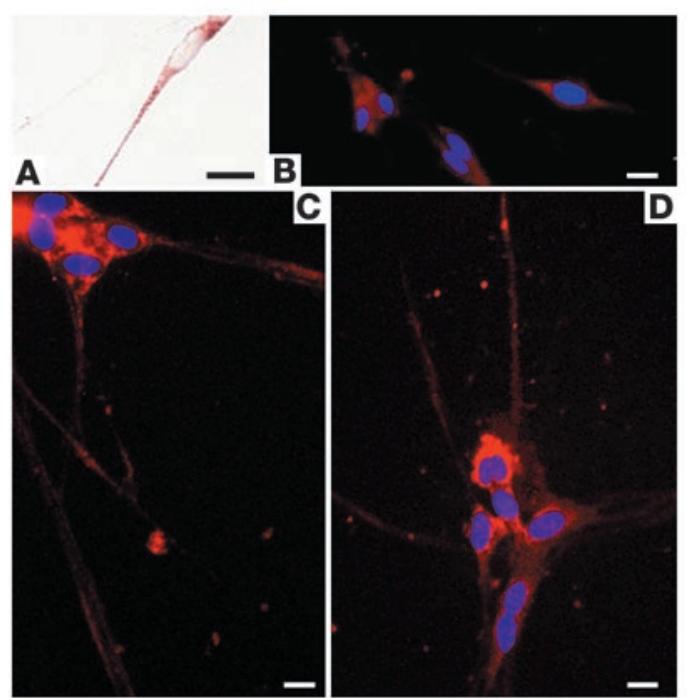

\section{Figure 11}

Primary cultures of guinea pig airway parasympathetic nerves stain positively with an antibody against eotaxin (A-D). Faint staining in untreated cells (B) was increased by treatment with IL-4 (C and D) or IL-13 (data not shown). Scale bars: $30 \mu \mathrm{m}$.

Measurement of $M 3$ receptor function. To test the sensitivity of airway smooth muscle to acetylcholine, M3 muscarinic receptors on the airway smooth muscle were stimulated using increasing doses of acetylcholine or methacholine (1-10 $\mu \mathrm{g} / \mathrm{kg}$, i.v.; Sigma-Aldrich), and bronchoconstriction was recorded. The vagus nerves were cut to eliminate any possible reflex induced by acetylcholine (36).

Bronchoalveolar lavage. At the end of the experiments, guinea pigs were killed by an overdose of pentobarbital. The lungs were lavaged with five $10-\mathrm{ml}$ aliquots of PBS. Lavage fluid was centrifuged at $300 \mathrm{~g}$ at $4^{\circ} \mathrm{C}$ for 10 minutes, and the cells were resuspended and counted on a hemocytometer. Cells were also cytospun onto glass slides, stained with Diff-Quik (VWR Scientific Products), and counted.

Immunohistochemical detection of eotaxin in airway nerves. At the conclusion of in vivo experiments, guinea pig lungs were excised, inflated with formalin, fixed for 4 hours, and processed as paraffin blocks. Sections were dewaxed, peroxidase quenched, and labeled with a 1:200 dilution of polyclonal antibody against eotaxin as previously described $(37,38)$ at room temperature. Preimmune rabbit serum was used as a negative control. Eotaxin expression was visualized using a streptavidin-HRP-linked system with 3,3'-diaminobenzidine. Human tracheas were obtained from organ donors and were provided by the Pacific Northwest Transplant Bank.

Histopathological evaluation of eosinophils. Nerves in the lungs were detected immunohistochemically in adjacent sections using a rabbit polyclonal antibody against PGP 9.5 (Biogenesis) as previously described (5). Briefly, formalin-fixed tissues were sectioned and stained at $4^{\circ} \mathrm{C}$ for 48 hours with primary antibody against PGP 9.5 diluted 1:4,000 in 1\% BSA in PBS. The secondary antibody, biotinylated goat anti-rabbit IgG (Vector Laboratories), was diluted 1:100 and incubated on the slides for 30 minutes at room temperature. Slides were rinsed with PBS and incubated with a streptavidin-linked HRP substrate (Vector Laboratories). The PGP 9.5 staining was visualized by incubating tissues with chromogen SG and nickel (Vector Laboratories), which stains airway nerves black. Lung sections were stained with antibody against PGP 9.5 (as described above) and then incubated in a $1 \%$ solution of chromotrope 2R (Sigma-Aldrich), which stains eosinophils red, for 30 minutes at room temperature. The slides were then washed in tap water, dehydrated in graded ethanol solutions, defatted in xylene, and permanently mounted.

The number of eosinophils within the walls of 4 different cartilaginous airways per animal were counted, using 5 animals per treatment group. The airways were first photographed with a video camera attached to a Nikon microscope, and the diameters and the areas of the airways were measured with MetaMorph software (version 6.1; Universal Imaging). Under oil immersion, the total number of eosinophils within the airway walls and below the basement membrane were counted in 10 consecutive high-power fields. In addition, eosinophils within $10 \mu \mathrm{m}$ of an airway nerve (approximately the width of 1 eosinophil) were also counted. Thus, the number of eosinophils per millimeter ${ }^{2}$ could be calculated for each treatment group, and the proportions of eosinophils associated (i.e., within 10 $\mu \mathrm{m}$ of a nerve) or not associated with airway nerves could be derived.

The $10-\mu \mathrm{m}$ limit used to define eosinophil association with nerves is approximately the average diameter of a normodense eosinophil. We have previously shown that eosinophil recruitment to the airway nerves occurs in antigen-challenged guinea pigs and in patients who have died of asthma (5). Released MBP is unlikely to diffuse far once in the extracellular matrix, because eosinophil MBP is cationic (pI 10.9), and it contains 5 unpaired cysteine residues per molecule, which readily form disulfide bonds. We therefore chose a distance to measure eosinophil association with airway nerves that is less than or equal to the width of an eosinophil.

Immunostaining of parasympathetic neurons cultured from guinea pig and from buman trachea and human neuroblastoma cells for eotaxin. Primary cultures of airway parasympathetic neurons were initiated from guinea pig tracheas and grown in serum-free medium for 1 week as previously described (39). Airway parasympathetic neurons were isolated from human trachea by the same method and stained for PGP 9.5 as described above. Primary cultured parasympathetic neurons from guinea pigs were fixed in methanol and acetone $(1: 1)$ for 3 minutes at room temperature 7 days after plating. Some were pre-
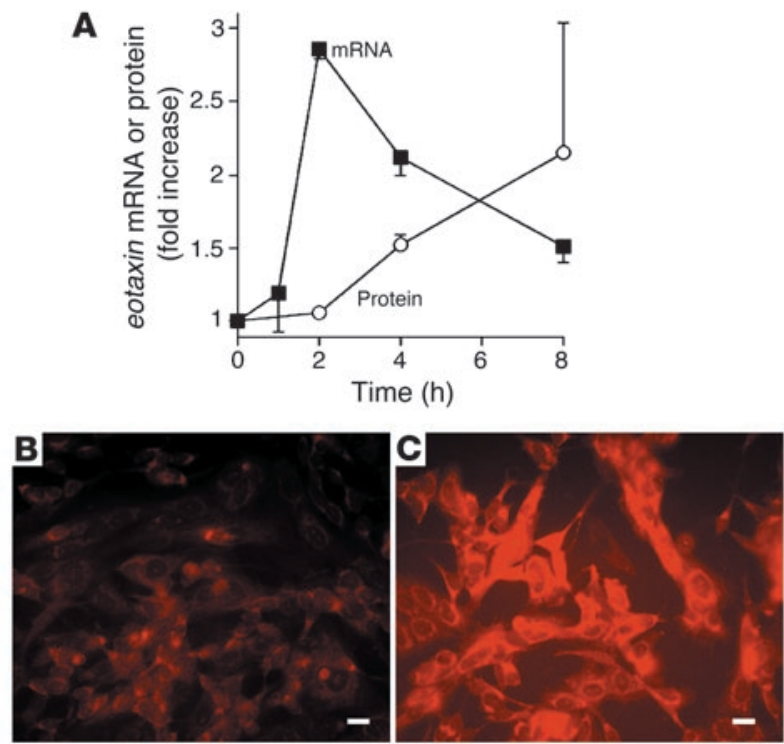

Figure 12

IL-4 stimulates expression of eotaxin in human neuroblastoma cells. eotaxin mRNA and protein expression were increased after exposure to IL-4 (1 ng/ml) (A). Immunostaining demonstrated increased eotaxin protein 8 hours after exposure to IL-4 (C) compared with untreated controls (B). Scale bars: $30 \mu \mathrm{m}$. 

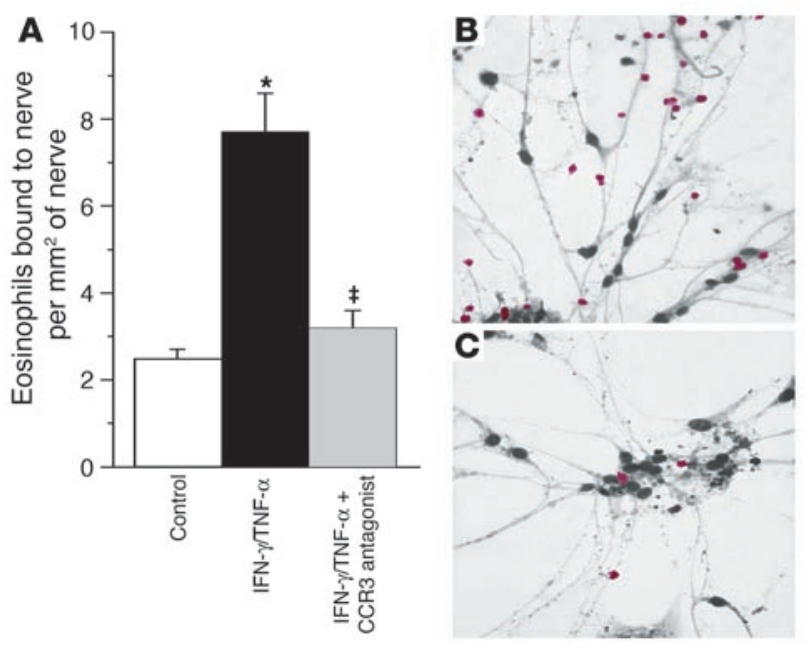

treated with $10 \mathrm{ng} / \mathrm{ml}$ recombinant human IL-4 (rhIL-4) for 4 or 8 hours before fixation. Cells were incubated with a 1:100 dilution of polyclonal antibody against eotaxin (37) at $4^{\circ} \mathrm{C}$ for 16 hours and then incubated with 1:500 diluted Texas Red-labeled anti rabbit IgG (Vector Laboratories) at $37^{\circ} \mathrm{C}$ for 2 hours. Preimmune rabbit serum was used as a negative control.

SK-N-SH cells were obtained from ATCC and grown in medium that contained $10 \%$ FCS. For eotaxin immunostaining, subconfluent cultures of SK-N-SH cells on glass chamber slides were fixed in methanol and acetone (1:1) for 3 minutes at room temperature after $2,4,8,16$, or 24 hours exposure to $1 \mathrm{ng} / \mathrm{ml} \mathrm{rhIL-4}$ (Chemicon International). Nonspecific binding of the primary antibody was blocked with normal goat serum. Cells were then incubated with anti-murine eotaxin primary antibody (PeproTech) diluted $1: 800$ at $4^{\circ} \mathrm{C}$ for 16 hours, followed by Texas red-labeled anti-rabbit IgG (Vector Laboratories) diluted 1:400 for 60 minutes at $37^{\circ} \mathrm{C}$. Negative control slides were generated using preimmune rabbit IgG (Vector Laboratories) as primary antibody.

All slides were mounted in aqueous medium with DAPI (Vector Laboratories) to stain the nuclei, examined by fluorescence microscopy (Nikon), and photographed using identical microscope and camera settings. Ten digital images per slide were taken to accurately reflect the overall staining. Experiments were performed in triplicate. Fluorescence intensity was quantified using MetaMorph software.

Immunostaining of cultured parasympathetic neurons and human neuroblastoma cells for IL-4 receptor. To detect IL-4 receptors, cultured SK-N-SH cells or guinea pig parasympathetic neurons were fixed in methanol and acetone (1:1) for 3 minutes at room temperature. Nonspecific binding of the primary antibody was blocked by incubation for 30 minutes with rabbit normal serum. Then cells were incubated with 1:200 dilution of goat antihuman IL-4 soluble receptor antibody (I6527; Sigma-Aldrich) at $4{ }^{\circ} \mathrm{C}$ for 16 hours, followed by fluorescein-labeled rabbit anti-goat antibody (Vector Laboratories) diluted 1:400 for another 60 minutes at $37^{\circ} \mathrm{C}$.

Expression of eotaxin mRNA. After stimulation with rhIL-4 or rhIL-13, total RNA was isolated using RNeasy Mini Kit (74106; QIAGEN). The RNA was reverse transcribed using SuperScript III (18080-051; Invitrogen Corp.) with random hexamer primers. Quantitative PCR was carried out in triplicate at $60^{\circ} \mathrm{C}$ over 45 cycles using Quantitect SYBR Green PCR kit (204143; QIAGEN). The amounts of PCR product were quantified using the Mx3000P real-time PCR system (Stratagene).

Oligonucleotide PCR primer pairs were designed from the published human sequences as follows: eotaxin-1: sense, AACCACCTGCTGCTTTAACC, antisense, TCCTGCACCCACTTCTTCTT; eotaxin-2: sense, GCCTTCTGTTCCTTGGTGTC, antisense, TGTACCTCTGGACCCACTCC; human

\section{Figure 13}

CCR3 antagonist inhibits eosinophil adhesion to primary cultures of parasympathetic nerves. Eosinophil adhesion to nerve cells was significantly increased in cells treated for 24 hours with IFN- $\gamma(1,000 \mathrm{U} / \mathrm{ml})$ and TNF- $\alpha$ (2 ng/ml) (A, black bar), compared with controls (A, white bar). The CCR3 antagonist significantly inhibited adherence of eosinophils to the nerves (A, gray bar). Representative photos of eosinophil (red) adhesion to nerves treated with IFN- $\gamma$ and TNF- $\alpha$ (IFN- $\gamma /$ TNF- $\alpha$ ) in the absence $(\mathbf{B})$ or presence $(\mathbf{C})$ of the CCR3 antagonist are shown. $n=4$. *Significantly different from control, $P=0.01$; \#significantly different from challenged, $P=0.04$. Scale bar: $20 \mu \mathrm{m}$.

eotaxin-3: sense, GGAGGAGTTTGGGAGAAACC, antisense, TGTGGCTGTATTGGAAGCAG; 18s rRNA: sense, GTAACCCGTTGAACCCCATT, antisense, CCATCCAATCGGTAGTAGCG. Threshold cycle number was measured, and the relative expression of eotaxin-1, -2, or -3 was adjusted for the threshold cycle for detection of 18s rRNA.

Isolation of guinea pig eosinophils. Guinea pigs were injected i.p. with a solution of $1 \%$ horse serum weekly for a period of 3 weeks to induce peritoneal eosinophilia. Twenty-four hours after the last treatment, they were anesthetized with xylazine $(10 \mathrm{mg} / \mathrm{kg}$, intramuscularly) and ketamine $(40 \mathrm{mg} / \mathrm{kg}$, intramuscularly), and peritoneal lavage was performed. The eosinophils were purified by centrifugation through a Percoll gradient (specific gravity, $1,090 \mathrm{~g} / \mathrm{ml}$ ), and red blood cells were removed by hypotonic lysis.

Coculture of guinea pigeosinophils and tracheal parasympathetic nerves. Eosinophils were coincubated with the cytokine-stimulated parasympathetic cholinergic nerves for 30 minutes $\left(5 \times 10^{4}\right.$ eosinophils/well). Nonadherent eosinophils were removed by washing 3 times with medium 199. The cells were then fixed with $3.7 \%$ (wt/vol) formaldehyde, and the eosinophils were detected using their endogenous peroxidase to catalyze a reaction, which in the presence of exogenous hydrogen peroxide yielded the red chromogen Vector Nova Red (Vector Laboratories). Nerves were counterstained with dilute hematoxylin.

Statistics. All data are expressed as mean \pm SE. Pilocarpine, frequency, acetylcholine, and methacholine responses were analyzed using 2-way ANOVA for repeated measures. Histological examination for tissue leukocytes and lavage leukocytes were analyzed by 2 -way ANOVA. A $P$ value of less than 0.05 was considered significant. All statistical analyses were made with Statview 4.5 software (Abacus Concepts).

\section{Acknowledgments}

This work was supported by NIH grants HL55543, HL10342, HL54659, and HL61013 and by US Department of Defense Grant DAMD17-02-1-0188. We thank Kirsten Blensly for performing some of the in vivo physiological experiments. We thank the members of the Pacific Northwest Transplant Bank, Portland, Oregon, for procuring and supplying tracheas from organ donors.

Received for publication April 21, 2005, and accepted in revised form October 25, 2005.

Address correspondence to: David B. Jacoby, Oregon Health and Science University, 3181 SW Sam Jackson Park Road, Portland, Oregon 97201, USA. Phone: (503) 494-0379; Fax: (503) 494-6670; E-mail: jacobyd@ohsu.edu. 
1. Fryer, A.D., and Maclagan, J. 1984. Muscarinic inhibitory receptors in pulmonary parasympathetic nerves in the guinea-pig. Br. J. Pharmacol. 83:973-978.

2. Minette, P., and Barnes, P.J. 1988. Prejunctional inhibitory muscarinic receptors on cholinergic nerves in human and guinea-pig airways. J. Appl. Physiol. 64:2532-2537.

3. Fryer, A.D., and Jacoby, D.B. 1992. Function of pulmonary M2 muscarinic receptors in antigen challenged guinea-pigs is restored by heparin and poly-l-glutamate. J. Clin. Invest. 90:2292-2298.

4. Minette, P.J., Lammers, J.W.J., Dixon, C.M.S., McCusker, M.T., and Barnes, P.J. 1989. A muscarinic agonist inhibits reflex bronchoconstriction in normal but not asthmatic subjects. J. Appl. Physiol. 67:2461-2465.

5. Costello, R.W., et al. 1997. Localization of eosinophils to airway nerves and effect on neuronal M2 muscarinic receptor function. Am. J. Physiol. 273:L93-L103.

6. Jacoby, D.B., Gleich, G.J., and Fryer, A.D. 1993. Human eosinophil major basic protein is an endogenous allosteric antagonist at the inhibitory muscarinic M2 receptor. J. Clin. Invest. 91:1314-1318.

7. Evans, C.M., Fryer, A.D., Jacoby, D.B., Gleich, G.J., and Costello, R.W. 1997. Pretreatment with antibody to eosinophil major basic protein prevents hyperresponsiveness by protecting neuronal M2 muscarinic receptors in antigen-challenged guinea pigs. J. Clin. Invest. 100:2254-2262.

8. Elbon, C.L., Jacoby, D.B., and Fryer, A.D. 1995. Pretreatment with an antibody to interleukin-5 prevents loss of pulmonary M2 muscarinic receptor function in antigen challenged guinea-pigs. Am.J. Respir. Cell Mol. Biol. 12:320-328.

9. Evans, C.M., Jacoby, D.B., and Fryer, A.D. 2001. Effects of dexamethasone on antigen-induced airway eosinophilia and $\mathrm{M}(2)$ receptor dysfunction. Am. J. Respir. Crit. Care Med. 163:1484-1492.

10. Jose, P.J., et al. 1994. Eotaxin: a potent chemoattractant cytokine detected in a guinea pig model of allergic airways inflammation. J. Exp. Med. 179:881-887

11. Yamada, H., et al. 2000. Eotaxin in induced sputum of asthmatics: relationship with eosinophils and eosinophil cationic protein in sputum. Allergy. 55:392-397.

12. Conroy, D.M., and Williams, T.J. 2001. Eotaxin and the attraction of eosinophils to the asthmatic lung. Respir. Res. 2:150-156.

13. Sawatzky, D.A., et al. 2002. Eosinophil adhesion to cholinergic nerves via ICAM-1 and VCAM-1 and associated eosinophil degranulation. Am. J. Physiol.
Lung Cell. Mol. Physiol. 282:L1279-L1288.

14. Adamko, D.J., Fryer, A.D., Bochner, B.S., and Jacoby, D.B. 2003. CD8+ T lymphocytes in viral hyperreactivity and $\mathrm{M} 2$ muscarinic receptor dysfunction. Am. J. Respir. Crit. Care Med. 167:550-556.

15. Adamko, D.J., Yost, B.L., Gleich, G.J., Fryer, A.D., and Jacoby, D.B. 1999. Ovalbumin sensitization changes the inflammatory response to subsequent parainfluenza infection. Eosinophils mediate airway hyperresponsiveness, $\mathrm{m}(2)$ muscarinic receptor dysfunction, and antiviral effects. J. Exp. Med. 190:1465-1478.

16. Costello, R.W., et al. 1999. Antigen-induced hyperreactivity to histamine: role of the vagus nerves and eosinophils. Am. J. Physiol. 276:L709-L714.

17. Chou, D.L., et al. 2005. Chronic aeroallergen during infancy enhances eotaxin-3 expression in airway epithelium and nerves. Am. J. Respir. Cell Mol. Biol. 33:1-8.

18. Yost, B.L., Gleich, G.J., and Fryer, A.D. 1999. Ozone induced hyperresponsiveness and blockade of M2 muscarinic receptors by eosinophil major basic protein. J. Appl. Physiol. 87:1272-1278.

19. Yost, B.L., Gleich, G.J., Jacoby, D.B., and Fryer, A.D. 2005. The changing role of eosinophils in long-term hyperreactivity following a single ozone exposure. Am. J. Physiol. Lung Cell. Mol. Physiol. 289:L627-L635.

20. Leckie, M.J., et al. 2000. Effects of an interleukin-5 blocking monoclonal antibody on eosinophils, airway hyper-responsiveness, and the late asthmatic response. Lancet. 356:2144-2148.

21. Flood-Page, P.T., Menzies-Gow, A.N., Kay, A.B., and Robinson, D.S. 2003. Eosinophil's role remains uncertain as anti-interleukin-5 only partially depletes numbers in asthmatic airway. Am. J. Respir. Crit. Care Med. 167:199-204.

22. Shen, H.H., et al. 2003. A causative relationship exists between eosinophils and the development of allergic pulmonary pathologies in the mouse. J. Immunol. 170:3296-3305.

23. Lee, J.J., et al. 2004. Defining a link with asthma in mice congenitally deficient in eosinophils. Science. 305: $1773-1776$.

24. Humbles, A.A., et al. 2004. A critical role for eosinophils in allergic airways remodeling. Science. 305:1776-1779.

25. Ying, S., et al. 1997. Enhanced expression of eotaxin and CCR3 mRNA and protein in atopic asthma. Association with airway hyperresponsiveness and predominant co-localization of eotaxin mRNA to bronchial epithelial and endothelial cells. Eur. J. Immunol. 27:3507-3516.

26. Holub, A., et al. 2003. Ligand density modulates eosinophil signaling and migration. J. Leukoc. Biol. 73:657-664.

27. Horie, S., et al. 1997. Intercellular adhesion molecule- 1 on eosinophils is involved in eosinophil protein $\mathrm{X}$ release induced by cytokines. Immunology. 90:301-307.

28. Mochizuki, M., Bartels, J., Mallet, A.I., Christophers, E., and Schroder, J.M. 1998. IL-4 induces eotaxin: a possible mechanism of selective eosinophil recruitment in helminth infection and atopy. J. Immunol. 160:60-68.

29. Heath, H., et al. 1997. Chemokine receptor usage by human eosinophils. The importance of CCR3 demonstrated using an antagonistic monoclonal antibody. J. Clin. Invest. 99:178-184.

30. Forssmann, U., Magert, H.J., Adermann, K., Escher, S.E., and Forssmann, W.G. 2001. Hemofiltrate CC chemokines with unique biochemical properties: HCC-1/CCL14a and HCC-2/CCL15. J. Leukoc. Biol. 70:357-366.

31. John, A.E., Thomas, M.S., Berlin, A.A., and Lukacs, N.W. 2005. Temporal production of CCL28 corresponds to eosinophil accumulation and airway hyperreactivity in allergic airway inflammation. Am. J. Pathol. 166:345-353.

32. Chiba, T., et al. 2005. RANTES and eotaxin enhance $\mathrm{CD} 11 \mathrm{~b}$ and CD18 expression on eosinophils from allergic patients with eosinophilia in the application of whole blood flow cytometry analysis. Int. Arch. Allergy Immunol. 137(Suppl. 1):12-16.

33. Sawatzky, D.A., et al. 2002. Eosinophil adhesion to cholinergic nerves via ICAM-1 and VCAM-1 and associated eosinophil degranulation. Am. J. Physiol. Lung Cell Mol. Physiol. 282:L1279-L1288.

34. Tachimoto, H., et al. 2000. CCR3-active chemokines promote rapid detachment of eosinophils from VCAM-1 in vitro. J. Immunol. 165:2748-2754.

35. Tachimoto, H., et al. 2002. Eotaxin-2 alters eosinophil integrin function via mitogen-activated protein kinases. Am. J. Respir. Cell Mol. Biol. 26:645-649.

36. Wagner, E.M., and Jacoby, D.B. 1999. Methacholine induces reflex bronchoconstriction. J. Appl. Physiol. 86:294-297.

37. Humbles, A.A., et al. 1997. Kinetics of eotaxin generation and its relationship to eosinophil accumulation in allergic airways disease: analysis in a guinea pig model in vivo. J. Exp. Med. 186:601-612.

38. Li, D., et al. 1997. Eotaxin protein and gene expression in guinea-pig lungs: constitutive expression and upregulation after allergen challenge. Eur. Respir.J. 10:1946-1954.

39. Fryer, A.D., et al. 1996. Cultures of airway parasympathetic nerves express functional $\mathrm{M}_{2}$ muscarinic receptors. Am. J. Respir. Cell Mol. Biol. 15:716-725. 